\title{
Capillary Surface Energy Elucidation of the Cosmic Dark Energy—Ordinary Energy Duality
}

\author{
Mohamed S. El Naschie \\ Department of Physics, Faculty of Science, University of Alexandria, Alexandria, Egypt \\ Email: $\underline{\text { Chaossf@aol.com }}$
}

Received 10 January 2014; revised 10 February 2014; accepted 18 February 2014

Copyright (C) 2014 by author and Scientific Research Publishing Inc. This work is licensed under the Creative Commons Attribution International License (CC BY). http://creativecommons.org/licenses/by/4.0/

c) (i) Open Access

\begin{abstract}
This short letter reports on an unsuspected and quite surprising connection between capillary forces and dark energy. We start with a very brief introduction of the role played by relativistic hydrodynamics in cosmic dark energy research, and then proceed from there to outline the proposed analogy between dark energy and non-relativistic effects of capillary surface energy.
\end{abstract}

\section{Keywords}

Dark Energy; Cantorian Space-Time; Relativistic Hydrodynamics; Capillary Surface Energy; Quantum Physics; Buckling of Elastic Shells; Imperfection Sensitivity

\section{Introduction}

In numerous previous publications, a fundamental theory was advanced to explain the baffling cosmic observation associated with conjectured dark energy and the surprising measured accelerated rather than decelerating expansion of the universe [1]-[4]. Exact calculations based on particle-wave duality in highly mathematical set theoretical formulation led to an ordinary measurable energy density of $E(O)=m c^{2} / 22$ where $m$ is the mass and $c$ is the speed of light, i.e. only 1/22 of Einstein's famous energy density [4]. This was a remarkable result and in full agreement with the latest and most accurate cosmic measurements and supernova analysis which incidentally led to the award of several Nobel Prizes in Physics on two different occasions [1] [4]. For dark energy the density found was $E(D)=m c^{2}(21 / 22)$ which amounts to exactly $m c^{2}-E(0)$ showing with absolute clarity that Einstein's density, lacking the quantum component, is blind to any distinction between ordinary energy and dark energy [1]-[4]. Thus apart of the quantitative resolution of this major problem, a fundamental conclusion was reached elevating Einstein's relativity formula $E=m c^{2}$ to a quantum relativity equation 
$E=\left(m c^{2} / 22\right)+m c^{2}(21 / 22)=m c^{2}$ where $E(\mathrm{O})$ is the ordinary energy of a quantum pre-particle in a five-dimensional Kaluza-Klein space time and $E(D)$ is the negative dark energy of the quantum pre-wave in the same Kaluza-Klein space-time [1]-[4]. Seen in this way we begin to understand why ordinary positive energy can be detected and measured while the negative dark energy could not, at least not directly nor using any conventional method [3] [4]. The reason for this failure is as simple as it is unexpected and is anchored in the deep logic of set theory [4]. In set theoretical terms, a quantum particle is a physical materialization of the zero set [3] [4]. The quantum wave on the other hand is the physical materialization of the empty set [3] [4]. Since "measurement" interferes with the empty set and causes it to become non-empty, the empty quantum "wave" set transmutes instantly to a zero quantum "particle" set at measurement [3] [4]. This is what we call wave collapse and that is why the negative dark energy of the wave cannot be measured in the ordinary way unless wave non-demolition measurements could be developed in the future [1]-[4].

The preceding set theoretical explanation, although mathematically and logically accessible and in some sense even intuitive, cannot be called physically obvious. For instance it is true that we have a clear picture of a particle with a wave as its cobordism, i.e. as its surface. Never the less particles and surfaces although inseparable, cannot be dealt with experimentally except via the contra-intuitive perspective of wave-particle duality [3] [4]. All the same it would be more than desirable to have a conjugate more down to earth and conventional physical picture to go hand in hand with the fundamental set theoretical interpretation just outlined [1]-[4].

\section{Relativistic Hydrodynamics, Dark Energy and Analogy with Capillary Surface Energy [4]-[7]}

In the present work we think that we have at long last found a parallel physical interpretation to our set theoretical picture which is in a one to one correspondence with the zero set-empty set particle-wave duality [3] [4], [7]-[9]. This we explain after a very short introduction to the role played by relativistic hydrodynamics in cosmic dark energy research [10].

\subsection{Relativistic Hydrodynamics and Dark Energy}

The total density parameter is easily deduced from Einstein's iconical leap of faith and genius $E=m c^{2}$ to be $\Omega$ (total $)=1$ and that is in full agreement with what Friedmann's equation ultimately predicts, namely [10]

$$
\Omega(\text { total })=\Omega_{\Lambda}+\Omega_{m}+\Omega_{k} \cong 0.685+0.315+0.000 .
$$

Never the less the most recent and most accurate cosmological measurements lead us to the unavoidable conclusion that $95.5 \%$ of $\Omega=1$ consists of dark matter $\Omega(\mathrm{dm})=0.26$ and $\Omega_{\Lambda}=0.69$ dark energy while only about $\Omega(\mathrm{b}) \cong 0.05$ is baryonic matter including radiation [3] [4] [10]. In this connection many theories were advanced to explain the mystery of dark energy [3] [4] [10]. The present author is of a reasonably firm opinion that the short coming of Einstein's relativity to reconcile theory and measurement is due to the non-physical character of Riemannian space-time in contrast to the subsequent theory of Einstein-Cartan and Cosserat-like space-time [11] [12]. For this reason we support the idea of using the theory of dark fluids and Chaplygin fluid because they point in the same general direction [10]-[12].

\subsection{Capillary Surface Energy and Dark Energy}

Let us consider a capillary surface which is something well known in fluid mechanics and in fact from various simple experiments which almost everyone encountered in elementary school physics [5]. On a fundamental level however the phenomena involves very complex nonlinearity effects and is related to the theory of a minimal surfaces [6]. The point is that the energy on the surface is meta-stable and is susceptible to spontaneous symmetry breaking bifurcation instability [7] by jumping into a much lower energy state similar in principle to phase transition as well as local buckling of thin walled structures [7], a field in which the present author was initially trained and specialized [7]. As we said earlier the subject is also closely related to minimal surfaces [6] and we note an almost esoteric property of capillary surfaces which is that although real, they have no thickness at all [5]. This is somehow an unexpected bridge between the pure mathematics of transfinite set theory and the real physics of capillary fluid mechanics [5] [8] [9]. We note further that despite the fact of being meta-stable, 
capillary surfaces are remarkably persistent in some experiments which make a good analogy to the steady state propagation of a quantum wave [8] [9]. Note also that the discrepancy between the measured $E=m c^{2} / 22$ and the theoretical $E=m c^{2}$ is reminiscent of the discrepancy between the theoretical buckling loads of elastic shells and its experimental magnitude which can be less than $1 / 3$ of the theoretical one [7].

\section{Conclusion}

To sum up we could look upon dark energy which is the negative energy of the quantum wave surface of the quantum particle core [8] as being analogous to the physically and classically real capillary surface energy which cannot always be easily measured due to spontaneous jump into the lower energy level of the core [5]-[7]. Here we are speaking of higher and lower in absolute terms and are of course disregarding the sign convention. We conclude by noting the immense importance of relativistic hydrodynamical models in physics and astrophysics [10] as well as the similarity between dark energy and the imperfection sensitivity of the buckling of thin elastic shell structures [7].

\section{Acknowledgements}

I am in more than one sense indebted to our teacher the late Prof. Dr. Dr. Theodor Lehmann who initiated my serious interest in Einstein's relativity although we were in a purely applied civil engineering mechanics department at the then Technical High School of Hannover, West Germany in the sixties of the last century. I am also indebted to Prof. J.M.T. Thompson (FRS) for introducing me to the book of H. Dingle "Science At The Crossroads" refuting special relativity though for the wrong reasons as it became obvious here. The discussion with Prof. Thompson also took place in a civil engineering department in University College, London, UK in 1969 with a stability research group which was established by Lord Henry Chilver.

\section{References}

[1] Linder, E. (2008) Dark Energy. Scholarpedia, 3, 4900.

[2] Sahni, V. (2003) Theoretical Models of Dark Energy. Chaos, Solitons \& Fractals, 16, 527-537. http://dx.doi.org/10.1016/S0960-0779(02)00221-7

[3] Helal, M.A., Marek-Crnjac, L. and He, J.-H. (2013) The Three Page Guide to the Most Important Results of M.S. El Naschie's Research in E-Infinity and Quantum Physics and Cosmology. Open Journal of Microphysics, 3, 141-145. http://dx.doi.org/10.4236/ojm.2013.34020

[4] Marek-Crnjac, L. (2013) An Invitation to El Naschie’s Theory of Cantorian Space-Time and Dark Energy. International Journal of Astronomy and Astrophysics, 3, 464-471. http://dx.doi.org/10.4236/ijaa.2013.34053

[5] Finn, R. (1999) Capillary Surface Interface. Notices of the American Mathematical Society, 46, 770-781.

[6] Dierkes, V., Hildebrandt, S., et al. (1992) Minimal Surfaces I. Springer Verlag, Berlin.

[7] El Naschie, M.S. (1990) Stress, Stability and Chaos in Structural Engineering: An Energy Approach. McGraw Hill, Int. Editions Civil Eng. Series, London, Tokyo.

[8] El Naschie, M.S. (2013) The Quantum Gravity Immirzi Parameter-A General Physical and Topological Interpretation. Gravitation and Cosmology, 19, 151-155. http://dx.doi.org/10.1134/S0202289313030031

[9] El Naschie, M.S. (2011) Quantum Entanglement as a Consequence of a Cantorian Micro Space-Time Geometry. Journal of Quantum Information Science, 1, 50-53. http://dx.doi.org/10.4236/jqis.2011.12007

[10] Rezzolla, L. and Zanotti, O. (2013) Relativistic Hydrodynamics. Oxford University Press, Oxford. http://dx.doi.org/10.1093/acprof:oso/9780198528906.001.0001

[11] El Naschie, M.S. (2014) Pinched Material Einstein Space-Time Produces Accelerated Cosmic Expansion. International Journal of Astronomy and Astrophysics, in Press.

[12] El Naschie, M.S. (2013) A Rindler-KAM Space-Time Geometry and Scaling the Planck Scale Solves Quantum Relativity and Explains Dark Energy. International Journal of Astronomy and Astrophysics, 3, 483-493. http://dx.doi.org/10.4236/ijaa.2013.34056 